Supporting Information

\title{
Modulating Optoelectronic Properties of Two-Dimensional Transition Metal Dichalcogenide Semiconductors by Photoinduced Charger Transfer
}

\author{
Jungwook Choi, Hanyu Zhang, and Jong Hyun Choi* \\ School of Mechanical Engineering, Purdue University \\ West Lafayette, Indiana 47907, United States \\ *Email: jchoi@purdue.edu
}

\section{Contents:}

1. PL spectra of TMDCs before and after annealing (Figure S1)

2. Effects of solvent on the optical characteristics of TMDCs (Figure S2 and S3)

3. Raman spectra of NiPc and MgPc (Figure S4)

4. XPS spectra of TMDCs with and without NiPc (Figure S5 and Table S1)

5. AFM, PL, and Raman studies on the TMDCs functionalized with naphthalene and F4TCNQ (Figure S6-S8)

6. Raman spectra of TMDCs and MPc-functionalized TMDCs (Figure S9)

7. PL quenching of multilayer WSe2 after NiPc functionalization (Figure S10)

8. AFM images and PL/Raman 2D profiles of MPc-functionalized TMDCs (Figure S11-13)

9. Static PL quenching of WSe2 with NiPc (Figure S14 and S15)

10. Analysis of $I-V$ curves measured using PC-AFM (Figure S16)

11. 2D current profiles and PL spectra of WSe2 and NiPc-functionalized WSe2 (Figure S17 and S18)

12. Photocurrent maps and PL spectra of MPc-functionalized TMDCs (Figure S19-S21)

13. References 


\section{PL spectra of TMDCs before and after annealing}
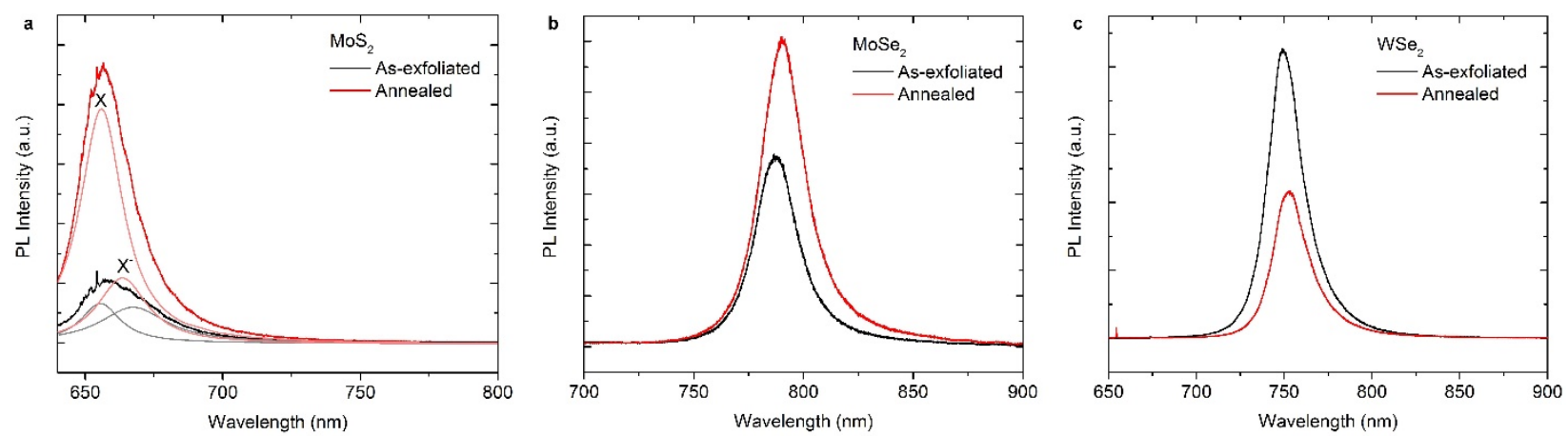

Figure S1. PL spectra of monolayer (a) $\mathrm{MoS}_{2}$, (b) MoSe2, and (c) WSe2 excited before and after annealing and subsequent air exposure. A 633-nm HeNe laser was used for excitation and measurements were performed at room temperature. The PL of TMDCs is dominated by exciton recombination. The $\mathrm{MoS}_{2}$ PL may be deconvoluted into two emission maxima of excitons (X) and negatively charged excitons (negative trions, $\mathrm{X}^{-}$) at room temperature as fitted by two Lorentzian functions in (a). For MoSez and WSe2, the charged exciton emission is observed only at low temperature. Thus, the formation of excitons and trions in emission spectra can be studied using $\mathrm{MoS}_{2}$ at the room temperature. The thermal annealing process may remove adsorbates and contaminants on the $\mathrm{MoS}_{2}$ layer. Once the $\mathrm{MoS}_{2}$ is exposed to ambient air after annealing, atmospheric molecules such as oxygen can interact with the fresh surface, depleting abundant electrons in $\mathrm{MoS}_{2}$ due to its strong electronegativity. ${ }^{1,2}$ This electron transfer results in suppression of formation of negative trions and thus increase exciton emission. Therefore, the intensity of overall PL spectra is enhanced with increased excitonic recombination (red curve in (a)). Similar PL enhancement is also observed in the $\mathrm{MoSe}_{2}$, which is another n-type electron-rich semiconductor, after annealing and air exposure. In contrast, p-type WSe 2 that lacks excessive electrons shows opposite behaviors as in (c). ${ }^{1}$ The decrease/increase in PL emission spectra before and after annealing implies the significant role of electrons that natively exist in the TMDCs, and thus most chemical doping methods rely on modulation of those excessive electrons for PL tuning. ${ }^{3-5}$ In this work, the excessive electrons in TMDCs are already transferred after annealing and air exposure, thereby enabling PL modulation via photoinduced charge transfer. Note that the annealing temperature was $250{ }^{\circ} \mathrm{C}$ which was lower than the temperature that would generate the defects in the TMDCs (e.g., $\left.500{ }^{\circ} \mathrm{C}\right) .{ }^{6}$ Therefore, the defects may not play a critical role in PL modulation in our study. 


\section{Effects of solvent on the optical characteristics of TMDCs}
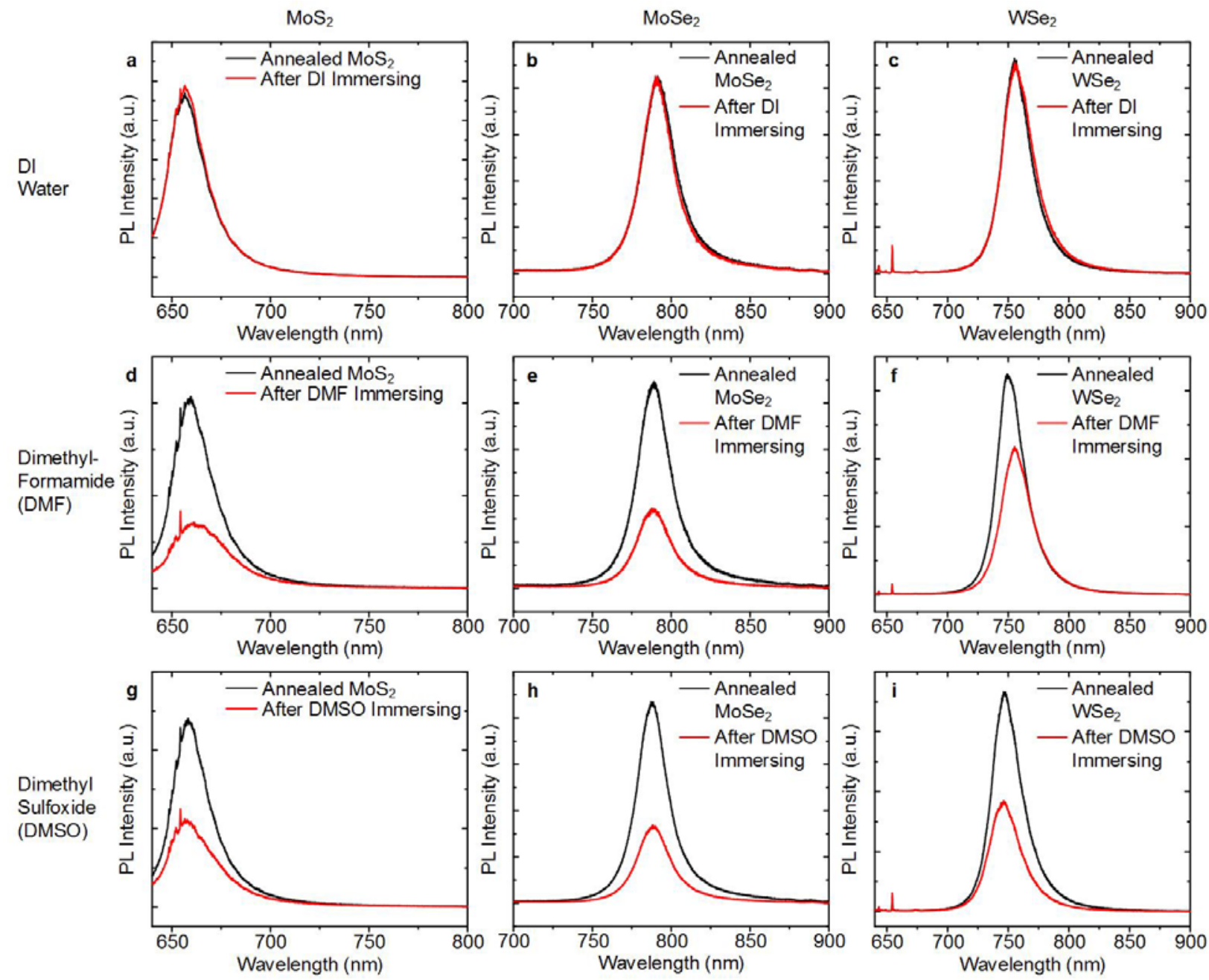

Figure S2. The PL spectra of $\mathrm{MoS}_{2}, \mathrm{MoSe}_{2}$, and WSe2 monolayers before and after immersion in (a-c) DI water, (d-f) dimethylformamide (DMF), and (g-i) dimethyl sulfoxide (DMSO). After mechanical exfoliation of TMDCs on the $\mathrm{SiO}_{2} / \mathrm{Si}$ substrate, all TMDC layers were annealed in argon at atmospheric pressure at $250{ }^{\circ} \mathrm{C}$ for $1 \mathrm{~h}$. Then, the TMDCs were immersed in DI water, DMF, or DMSO for 10 min, followed by rinsing with DI water and blow-drying with air. While DI water has little effects on the PL of TMDCs $(a-c)$, DMF and DMSO quench the PL of TMDCs significantly $(\mathrm{d}-\mathrm{i})$, likely due to the methyl group $\left(-\mathrm{CH}_{3}\right)$ in the solvents that attracts electrons. As a result, the carrier density in the TMDC layers changes and excitonic recombination is suppressed. In order to exclude any possible solvent effects, we used only DI water for dissolving MPcs throughout the work. 

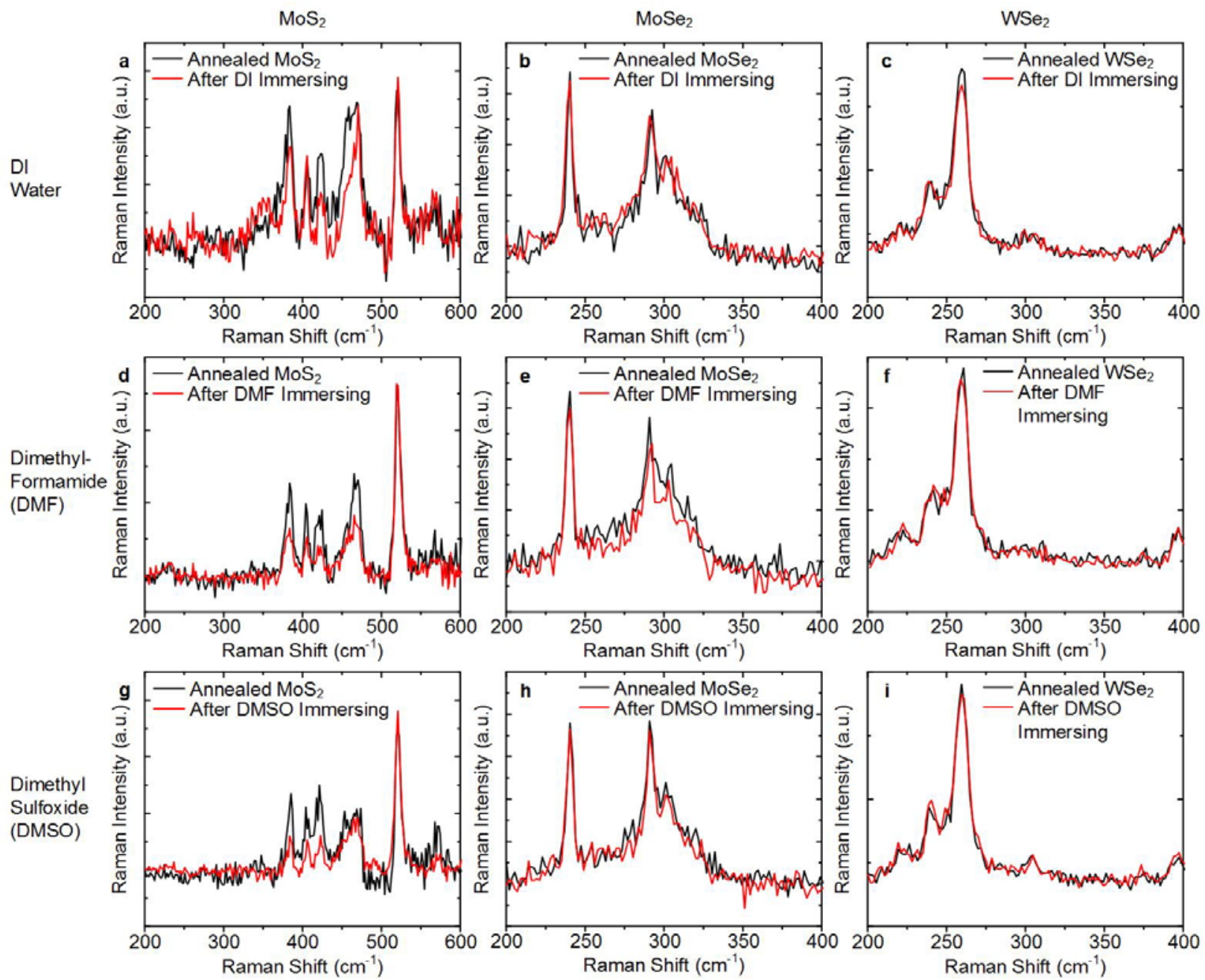

Figure S3. The Raman spectra of $\mathrm{MoS}_{2}, \mathrm{MoSe}_{2}$, and WSe2 before and after immersion in (a-c) DI water, $(d-f) D M F$, and (g-i) DMSO. In contrast to the PL, there is no significant change in the Raman signatures after immersion in the solvents. 


\section{Raman spectra of NiPc and MgPc}
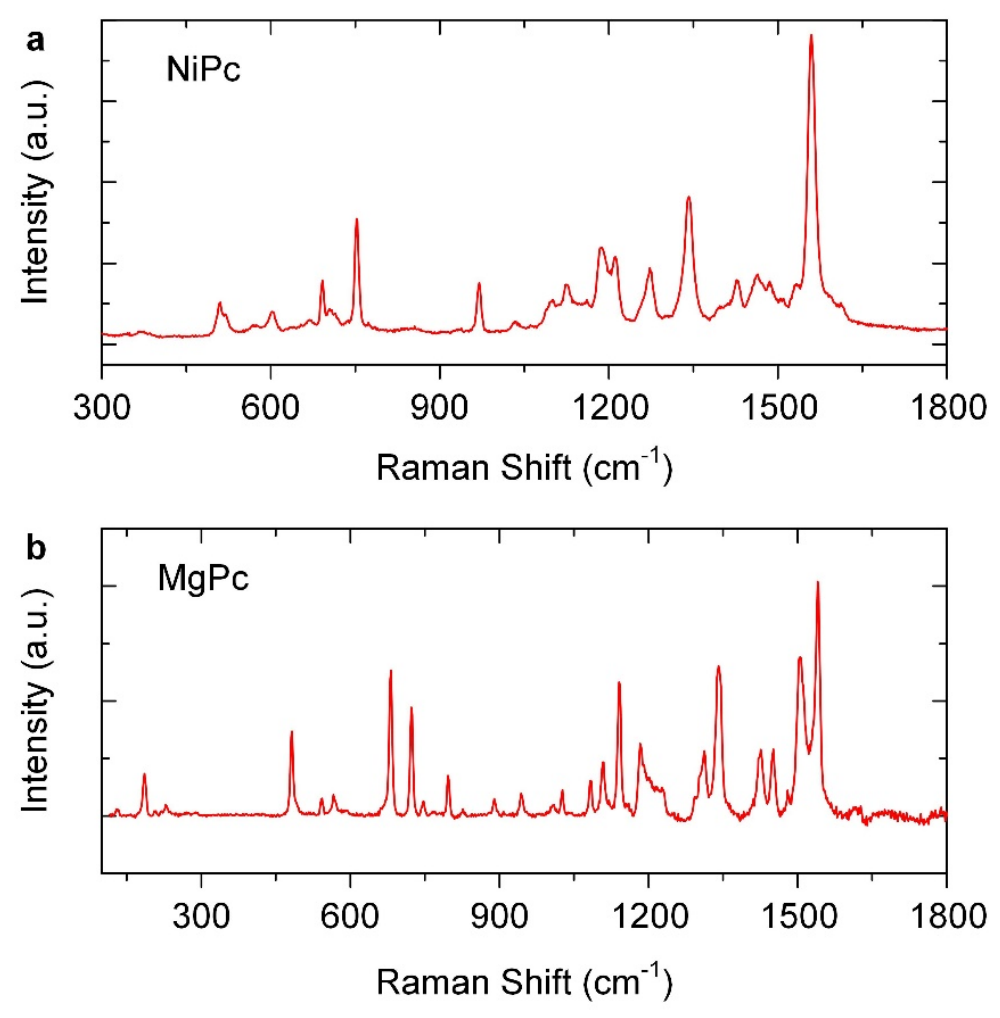

Figure S4. Raman spectra of (a) NiPc and (b) MgPc (with $633 \mathrm{~nm}$ excitation) show several signatures. A 10- $\mu \mathrm{L}$ aliquot of $1 \mathrm{mM}$ NiPc or the MgPc solution was drop-casted on the surface of $\mathrm{SiO}_{2}$, and the Raman spectra were collected after DI water evaporation (after 1 hour). The pathalocyanine signatures are consistent with previous reports. ${ }^{7,8}$ 


\section{XPS spectra of TMDCs with and without NiPc}
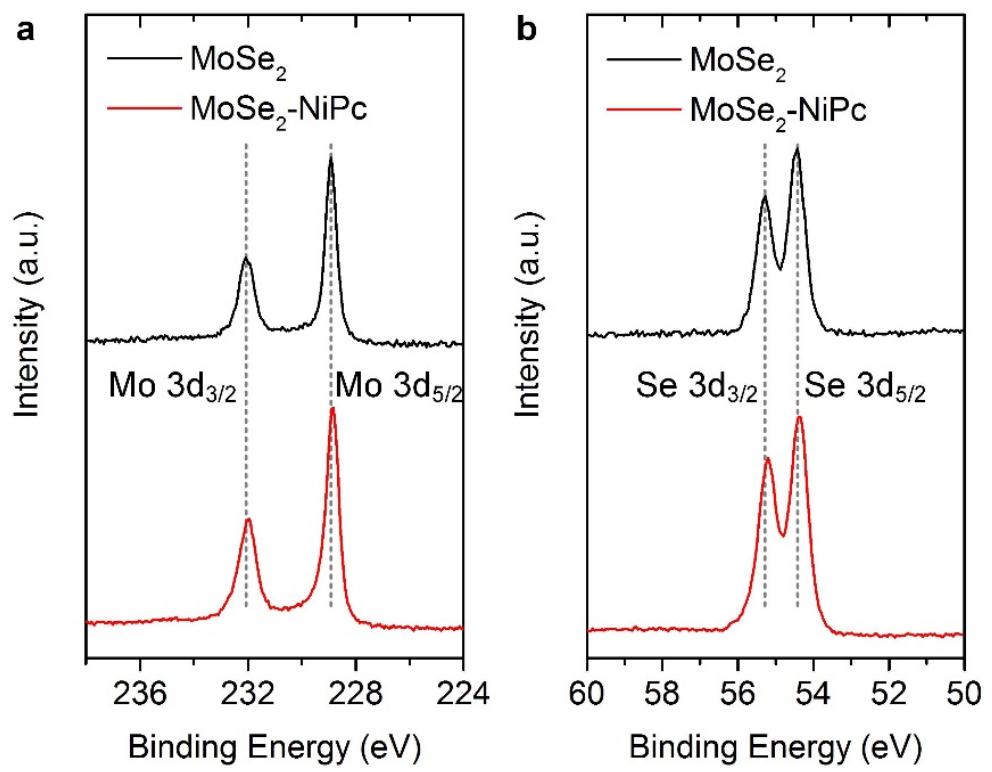

Figure S5. Mo 3d and S 2p core level XPS spectra of MoSe2 with and without NiPc, showing little changes after functionalization. 


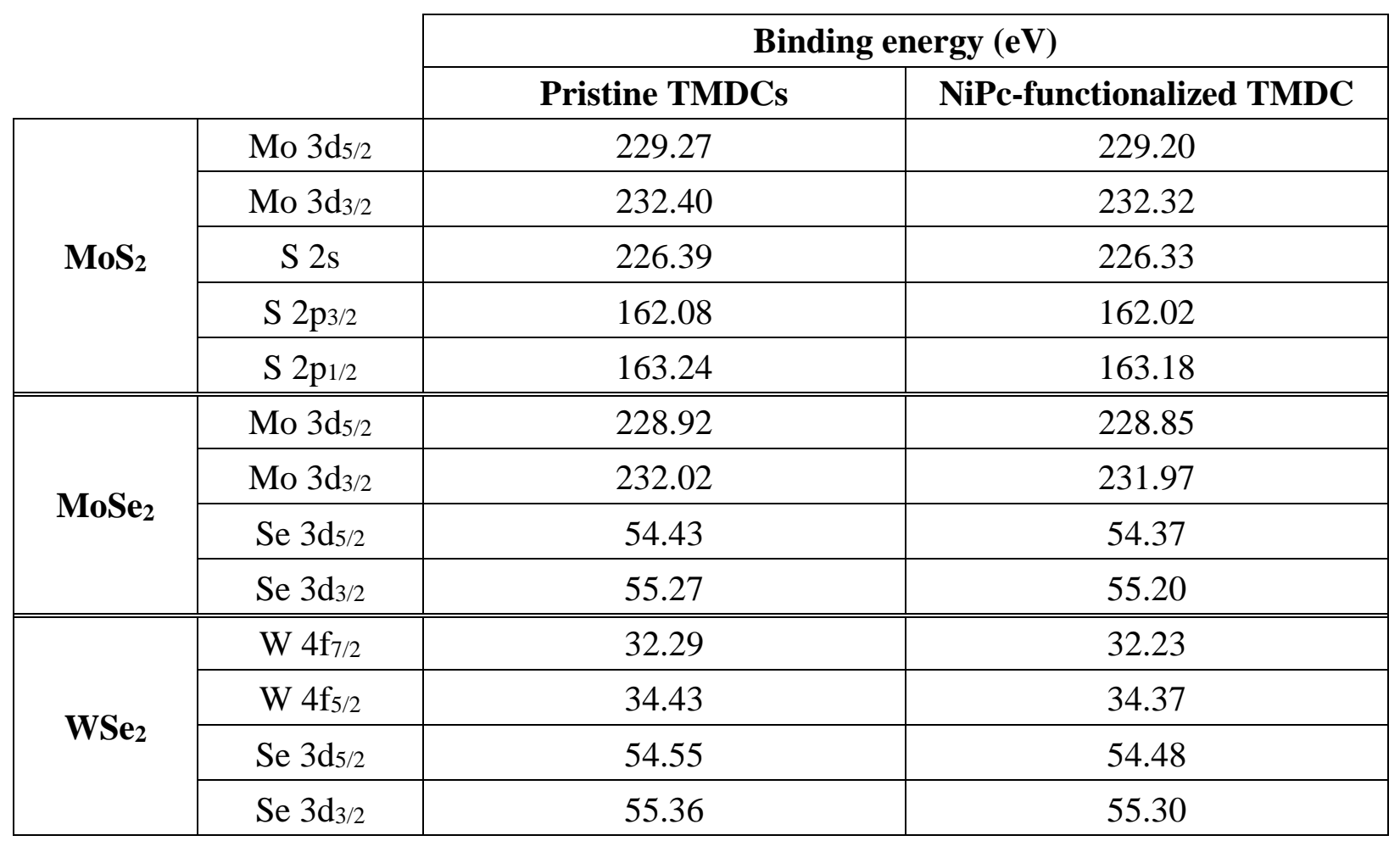

Table S1. Summary of XPS binding energies of $\mathrm{MoS}_{2}, \mathrm{MoSe}_{2}$, and WSe2 with and without NiPc. A shift of binding energy in XPS spectra would indicate the offset of Fermi level, which may be used as a measure of determining the extent of doping in the TMDCs. ${ }^{9}$ Previous studies reported that chemical doping by surface charge transfer results in the binding energy shift in the range of 0.3-0.8 eV. ${ }^{5,9,10}$ Our XPS spectra reveal minimal doping effects with binding energy shifts less than $0.07 \mathrm{eV}$. We observe a slight deviation in binding energies of Mo 3d and Se 3d between $\mathrm{MoS}_{2}$ and MoSe2 $(\sim 0.35 \mathrm{eV})$ and between MoSe2 and WSe2 $(\sim 0.1 \mathrm{eV})$. This discrepancy could be due to local variations in stoichiometry and defects, resulting in different XPS spectra even in the same flake. ${ }^{11,12}$ 


\section{AFM, PL, and Raman studies on the TMDCs functionalized with naphthalene and F $_{4}$ TCNQ}

a
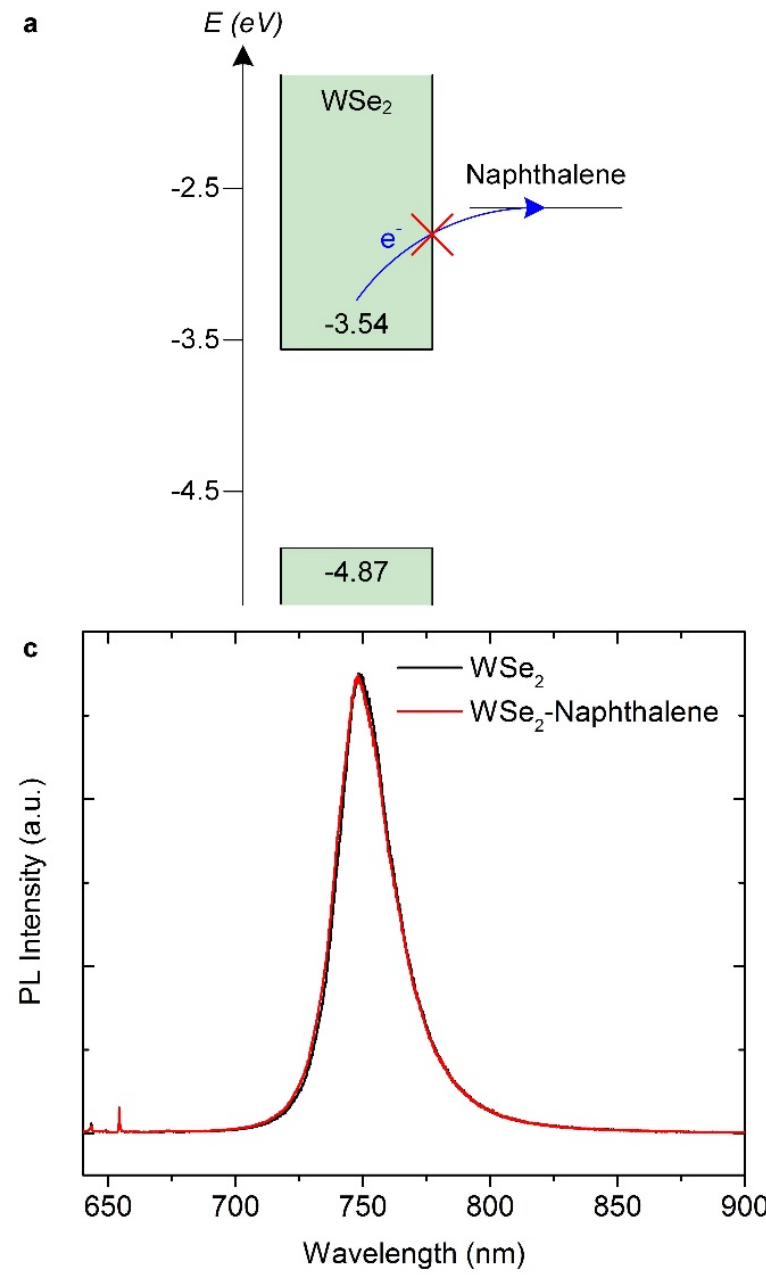

b
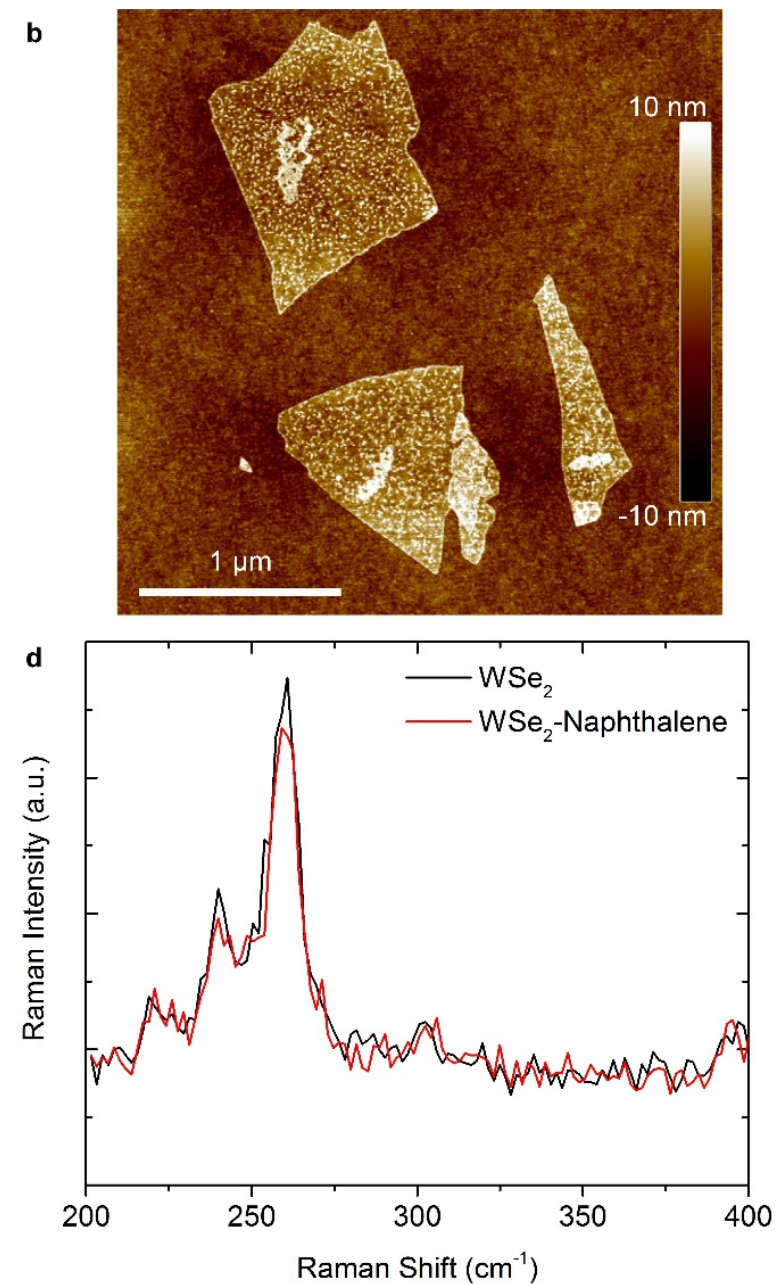

Figure S6. AFM, PL, and Raman characterization of naphthalene derivative-functionalized monolayer WSe2. Approximately $100 \mu \mathrm{M}$ sodium 2-naphthalenesulfonate (NS) was dissolved in DI water, and annealed WSez flakes were subsequently submerged in the solution for $20 \mathrm{~min}$. Then, the sample was rinsed by DI water and dried by air. (a) Relative energy offset between the band edges of monolayer WSez and the typical reduction potential of naphthalene derivatives. The reduction potential of NS is unknown to the best of our knowledge, but it should be positioned well above CBM of WSe2. For example, the reduction potential of naphthalene-1,5-disulfonate and naphthalene-1,2-dimethyl is around -2.59 and $-2.23 \mathrm{eV}$, respectively. ${ }^{13}$ Therefore, the photoinduced charge transfer from the WSe 2 to naphthalene is not energetically favorable. (b) AFM height image of NS-functionalized WSe 2 flakes. (c) PL spectra of pristine WSe 2 and NSfunctionalized WSe2. Because excited electrons in the WSe 2 are not allowed to transfer to NS, excitonic recombination is not altered, resulting in no change of PL. (d) Raman spectra of WSe2 before and after NS functionalization, showing nearly identical signatures. It is noted that the pristine WSez flake was immersed in DI water without NS for 20 min and dried before PL and Raman measurement as a control. The results support the photoinduced charge transfer mechanism in MPc-functionalized TMDCs. 

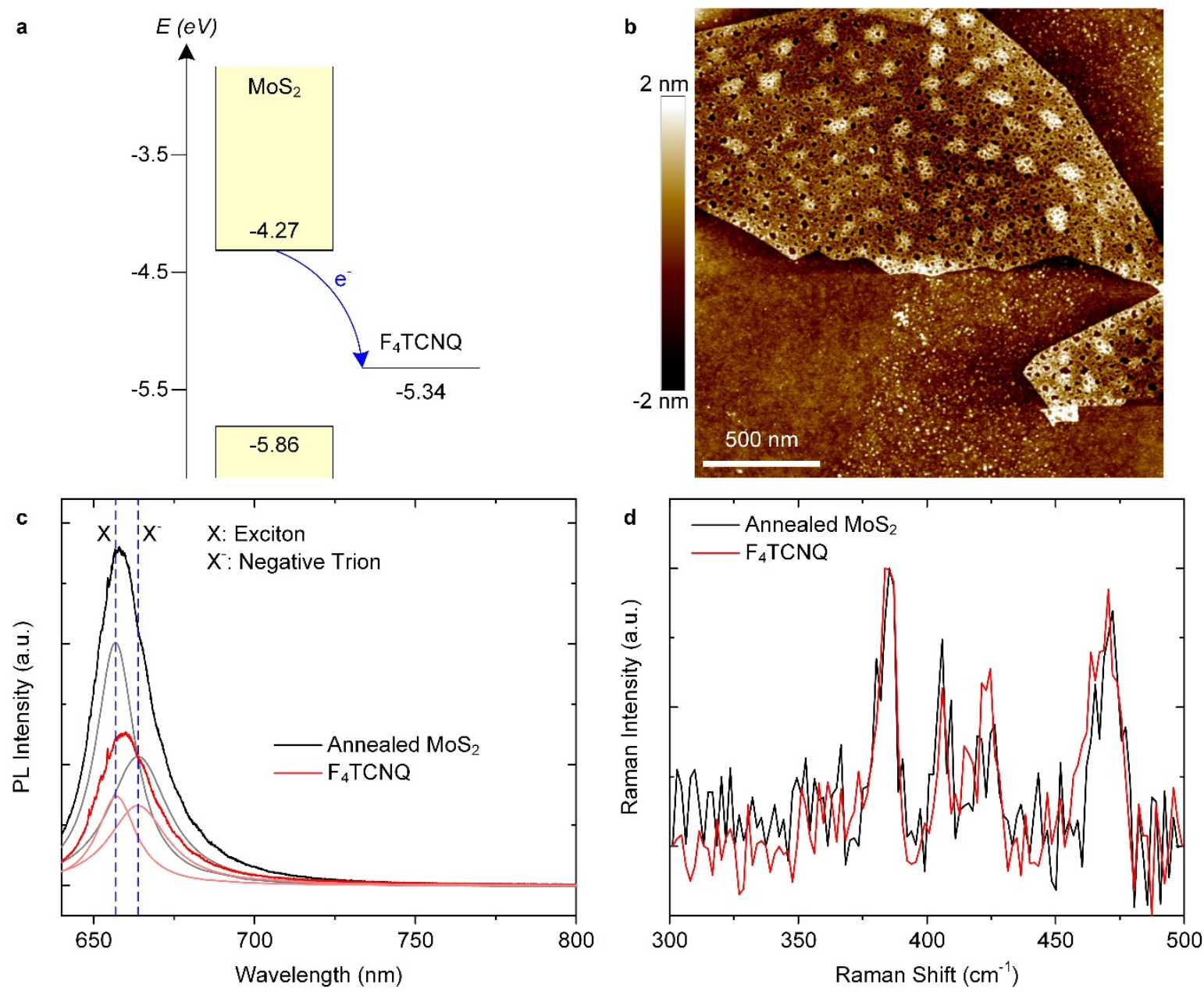

Figure S7. AFM, PL, and Raman characterization of monolayer $\mathrm{MoS}_{2}$ functionalized with 2,3,5,6tetrafluoro-7,7,8,8-tetracyanoquinodimethane (F4TCNQ). Approximately $1 \mathrm{mM} \mathrm{F} 4 \mathrm{TCNQ}$ was dissolved in DI water, and an annealed $\mathrm{MoS}_{2}$ layer was incubated in the solution for $20 \mathrm{~min}$, followed by DI water rinsing and air-drying. (a) The reduction potential of F4TCNQ is located between the band edges of $\mathrm{MoS}_{2},{ }^{3,4}$ such that photoexcited electrons can be transferred from $\mathrm{MoS}_{2}$ to F4TCNQ. (b) AFM height image of F4TCNQ-functionalized MoS 2. (c) PL spectra of pristine $\mathrm{MoS}_{2}$ and $\mathrm{F}_{4} \mathrm{TCNQ}$-functionalized $\mathrm{MoS}_{2}$. Due to the photoinduced charge transfer, exciton recombination is diminished, resulting in PL quenching. The intensity of exciton peak is significantly decreased after functionalization, and spectral weight of exciton (intensity of exciton emission divided by total intensity of PL) decreases from 0.72 to 0.58 . This result is contrast to a previous report, ${ }^{3}$ where the exciton emission increased after F4TCNQ functionalization by the transfer of excessive electrons and the resulting decrease of negative trion formation. In our experimental conditions, the abundant electrons $\mathrm{MoS}_{2}$ are depleted through annealing and air exposure before functionalization, and thus, photoinduced charge transfer mechanism can dominate the PL response. (d) Raman spectra of $\mathrm{MoS}_{2}$ before and after F4TCNQ functionalization, showing nearly identical signatures. It is noted that the pristine $\mathrm{MoS}_{2}$ was immersed in DI water without F4TCNQ for 20 min and dried before PL and Raman measurements as a control. 


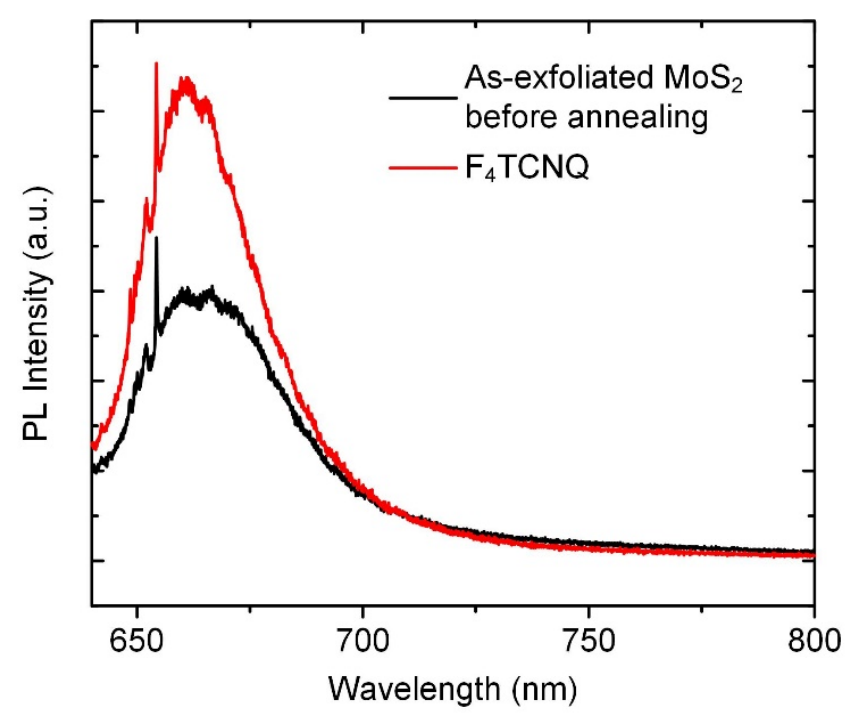

Figure S8. PL spectra of as-exfoliated $\mathrm{MoS}_{2}$ and $\mathrm{F}_{4} \mathrm{TCNQ}$-functionalized $\mathrm{MoS}_{2}$. In contrast to the annealed $\mathrm{MoS}_{2}-\mathrm{F}_{4} \mathrm{TCNQ}$ in Figure S7 that shows the PL quenching, the PL intensity of asexfoliated $\mathrm{MoS}_{2}$ increases after functionalization of $\mathrm{F}_{4} \mathrm{TCNQ}$. It is attributed to the depletion of excessive electrons and thereby decreased formation of negative trions, which is consistent with previous report. ${ }^{3}$ The opposite emission behaviors with the same functional molecules indicate the importance of initial state of TMDCs. In our experiments, the PL modulation is determined by the amount of excessive electrons in the native TMDCs. 


\section{Raman spectra of TMDCs and MPc-functionalized TMDCs}
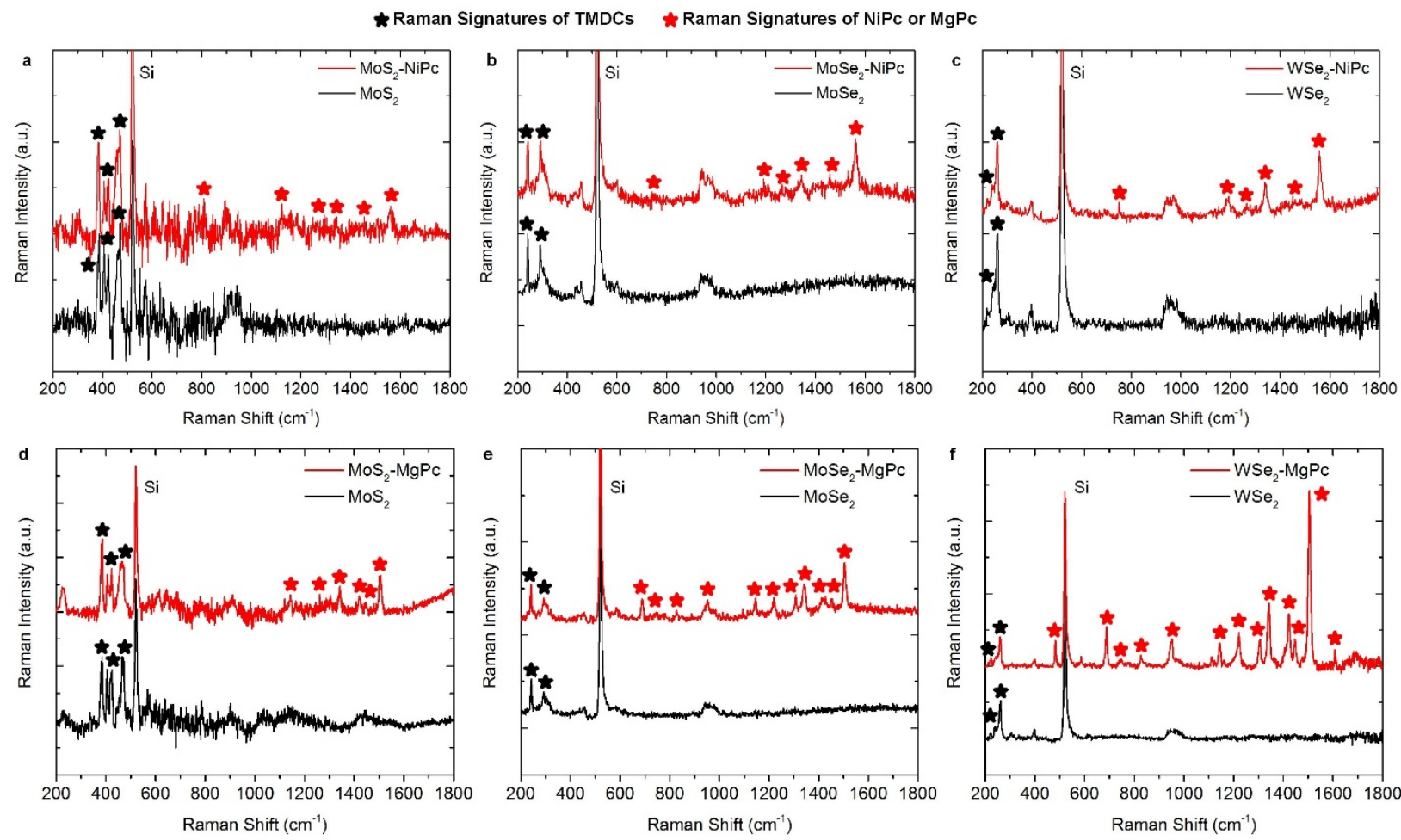

Figure S9. Raman spectra of (a) $\mathrm{MoS}_{2}$ and $\mathrm{MoS}_{2}-\mathrm{NiPc}$, (b) MoSe2 and MoSe2-NiPc, (c) WSe2 and WSe2-NiPc, (d) MoS2 and MoS2-MgPc, (e) MoSe2 and MoSe2-MgPc, and (f) WSe2 and WSe2MgPc. Black stars indicate the Raman modes of the TMDCs, and red stars represent the Raman signatures of the MPcs. Both spectra of pristine TMDCs and MPc-functionalized TMDCs are collected from the same flakes before and after functionalization, indicating successful functionalization. 


\section{PL quenching of multilayer WSe $\mathbf{e}_{2}$ after NiPc functionalization}
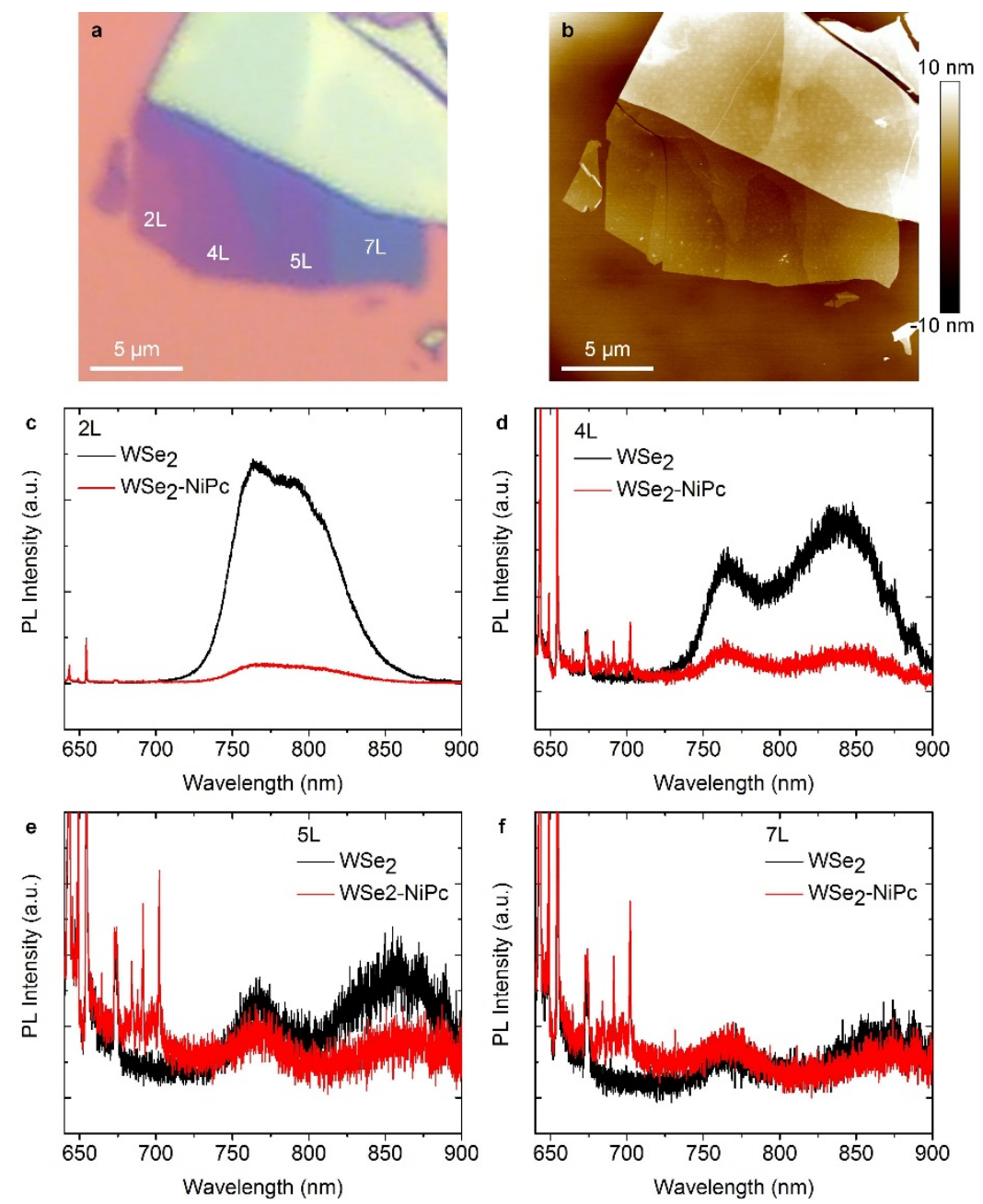

Figure S10. PL spectra of WSe2 with various thickness ranging from 2 to 7 layers before and after NiPc functionalization. (a) Optical microscope image of the WSe2 flake. (b) AFM height image of the same WSe2 flake shown in (a). (c-f) PL spectra of 2L (c), 4L (d), 5L (e), and 7L (f) WSe 2 before and after functionalization. In contrast to monolayer WSe2 where CBM and VBM coincide at the $\mathrm{K}$ point, the multilayer WSe 2 has upshifted valence band at the $\Gamma$ point and downshifted conduction band at the $\Lambda$ point. ${ }^{14}$ The local shift of conduction and valence bands makes them indirect bandgap semiconductors. The emission from direct (indirect) bandgap transitions are located at 759.7 (791.8), 767.6 (836.1), 767.7 (857.6), and $767.7 \mathrm{~nm}(872.6 \mathrm{~nm})$ for 2L, 4L, 5L and 7L, respectively. After NiPc functionalization, the PL quenches regardless of origin of emission due to photoinduced excited electron transfer from the WSez to the NiPc. It is noted that several Raman signatures from NiPc are also shown in (c-f) after functionalization, which are located at 702.2 (1561.8), 691.6 (1343.6), 688.1 (1270), 684 (1182.9), and $664.5 \mathrm{~nm}\left(753.9 \mathrm{~cm}^{-1}\right)$. 


\section{AFM image, PL and Raman maps of MPc-functionalized TMDCs}
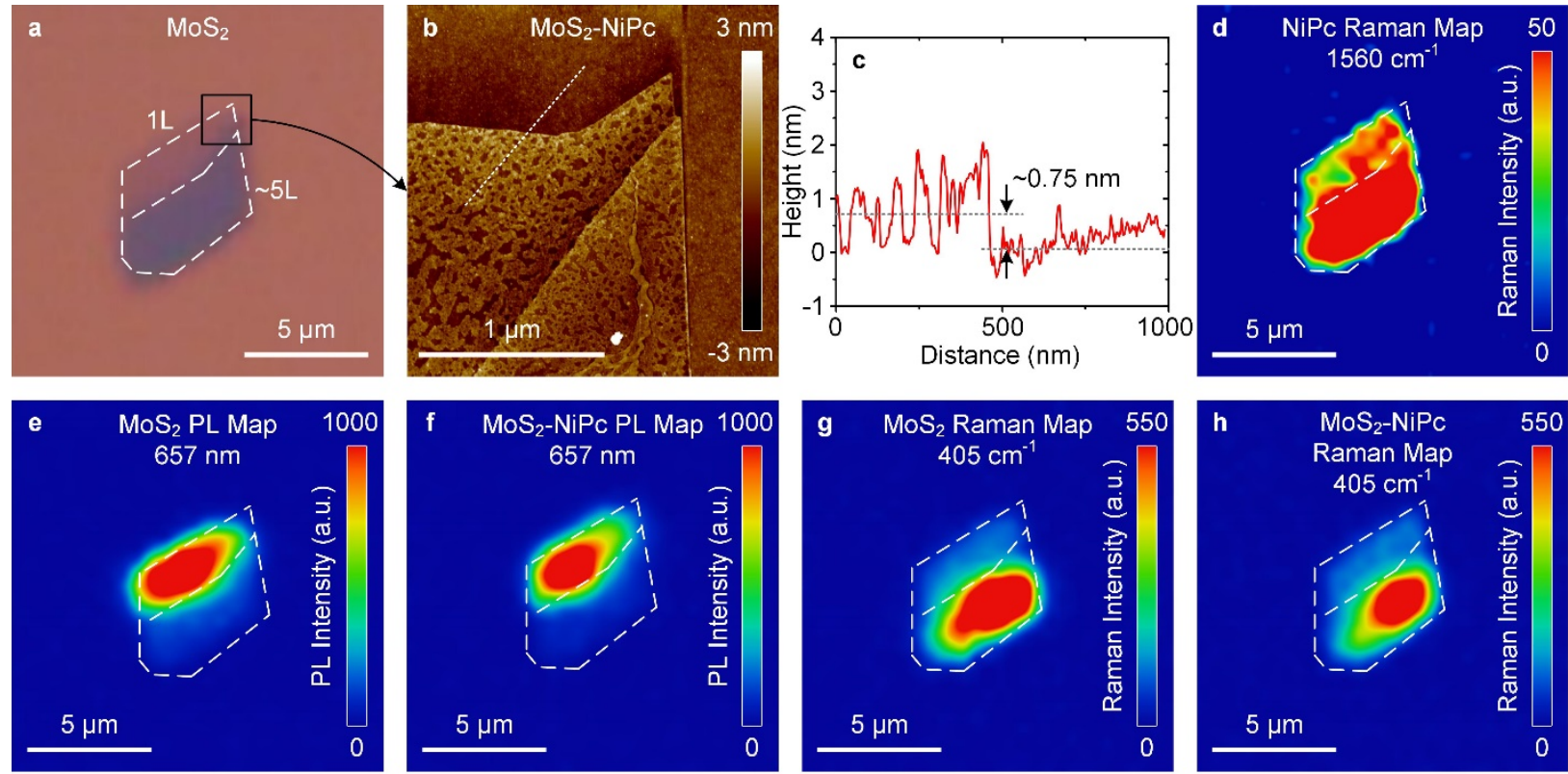

Figure S11. Characterizations of $\mathrm{MoS}_{2}$ before and after NiPc functionalization. (a) Optical microscope image of $1 \mathrm{~L}$ and $5 \mathrm{~L} \mathrm{MoS}$. (b) AFM image measured after NiPc functionalization. (c) AFM height profile obtained along the white dotted line in (b). (d) Raster-scanned Raman intensity map of NiPc at $1560 \mathrm{~cm}^{-1}$ corresponds the shape of the $\mathrm{MoS}_{2}$ flake, indicating selective and uniform functionalization of NiPc on MoS2. (e,f) PL intensity map of $\mathrm{MoS}_{2}$ at $657 \mathrm{~nm}$ before (e) and after (f) NiPc functionalization. No significant PL quenching after functionalization supports the photoinduced charge transfer mechanism where the photoexcited electrons in $\mathrm{MoS}_{2}$ are not allowed to transfer to the NiPc. (g,h) Raman intensity map of MoS 2 at $405 \mathrm{~cm}^{-1}$ before (g) and after (h) NiPc functionalization, showing no change in the Raman signature. 

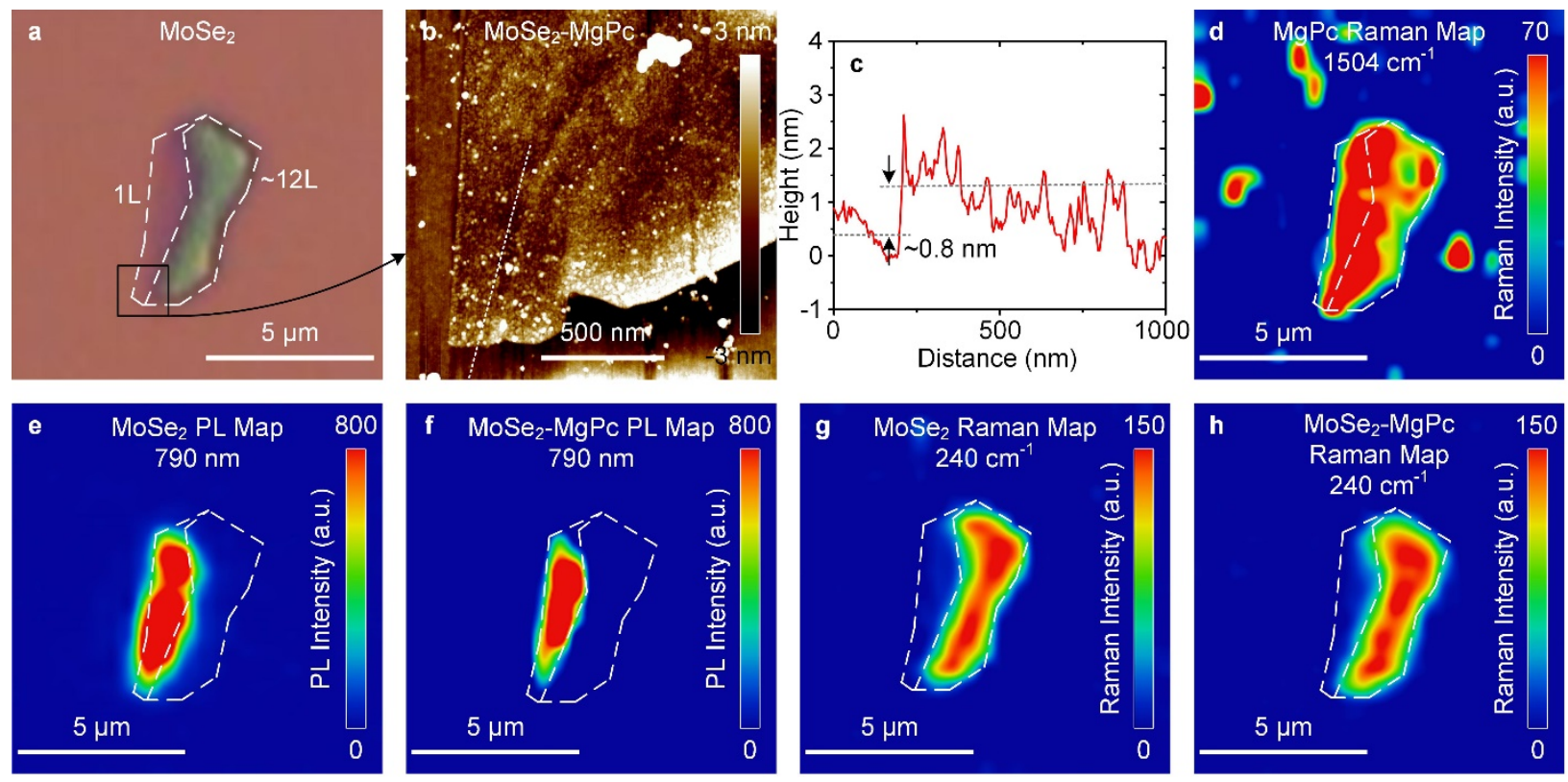

Figure S12. Characterization of $\mathrm{MoSe}_{2}$ before and after MgPc functionalization. (a) Optical microscope image of $1 \mathrm{~L}$ and 12L MoSe2. (b) AFM image measured after MgPc functionalization. (c) AFM height profile obtained along the white dotted line in (b). (d) Raster-scanned Raman intensity map of MgPc at $1504 \mathrm{~cm}^{-1}$ corresponds the shape of the MoSez flake. Due to relatively low water solubility of MgPc, several MgPc aggregates are found on the surface of $\mathrm{SiO}_{2}$. (e,f) PL intensity map of $\mathrm{MoSe}_{2}$ at $790 \mathrm{~nm}$ before (e) and after (f) MgPc functionalization. No significant PL quenching after functionalization supports the photoinduced charge transfer mechanism where the photoexcited electrons in MoSez are not allowed to transfer to the MgPc. (g,h) Raman intensity map of MoSez at $240 \mathrm{~cm}^{-1}$ before (g) and after (h) MgPc functionalization, showing no change in the Raman signature. 

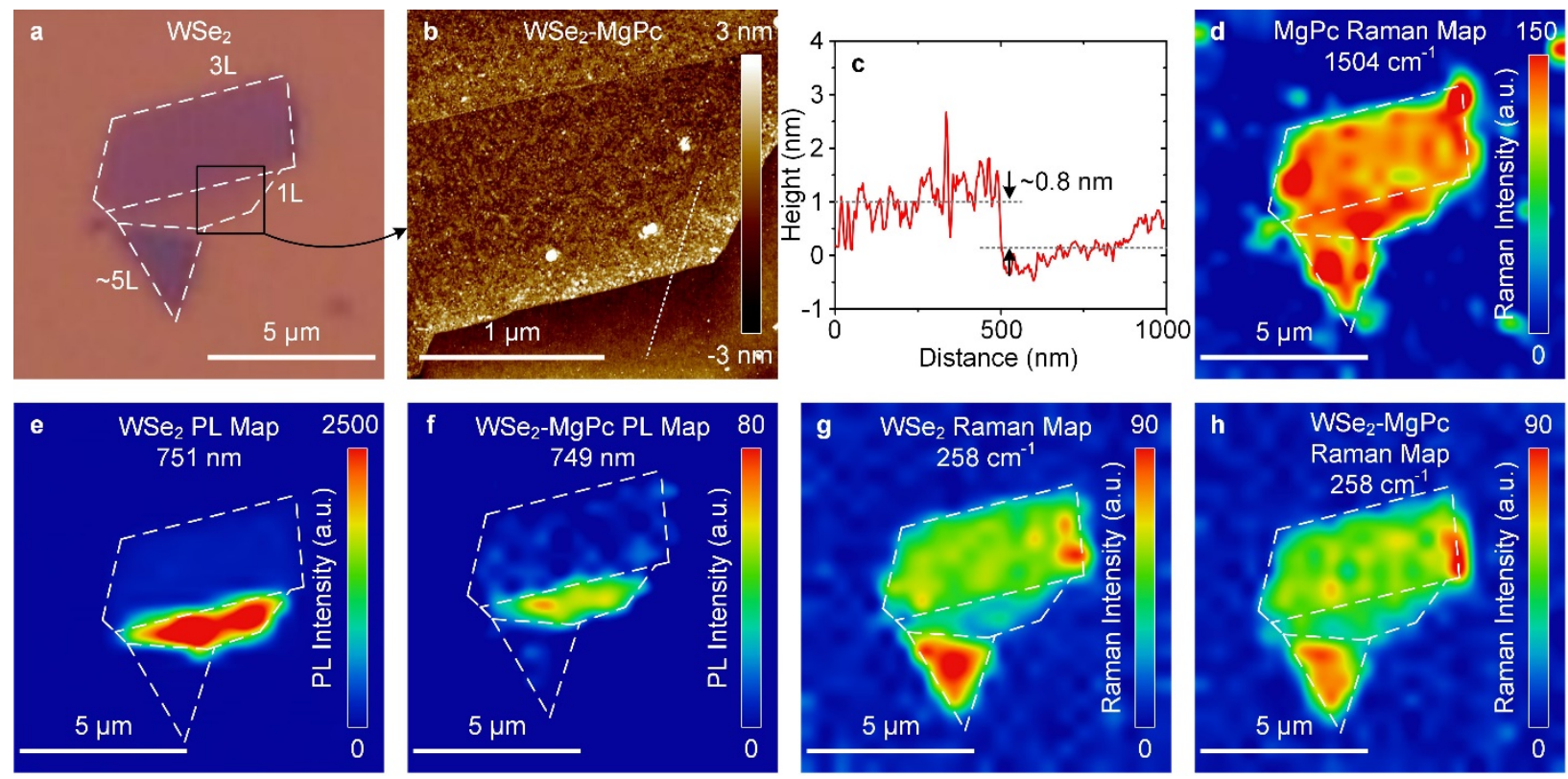

Figure S13. Characterization of WSe2 before and after MgPc functionalization. (a) Optical microscope image of 1L, 3L and 5L WSe2. (b) AFM image measured after MgPc functionalization. (c) AFM height profile obtained along the white dotted line in (b). (d) Raster-scanned Raman intensity map of MgPc at $1504 \mathrm{~cm}^{-1}$ corresponds the shape of the WSez flake. (e,f) PL intensity map of WSe2 before (at $751 \mathrm{~nm}$ ) (e) and after (at $749 \mathrm{~nm}$ ) (f) MgPc functionalization. The WSe 2 $\mathrm{PL}$ is quenched and blue-shifted after MgPc functionalization. Because the photoexcited electrons in WSez are allowed to transfer to the MgPc, the recombination diminishes, resulting in significant PL quenching. (g,h) Raman intensity map of WSe2 at $258 \mathrm{~cm}^{-1}$ before (g) and after (h) MgPc functionalization, showing no change in Raman signature. 


\section{Static PL quenching of $\mathrm{WSe}_{2}$ with NiPc}

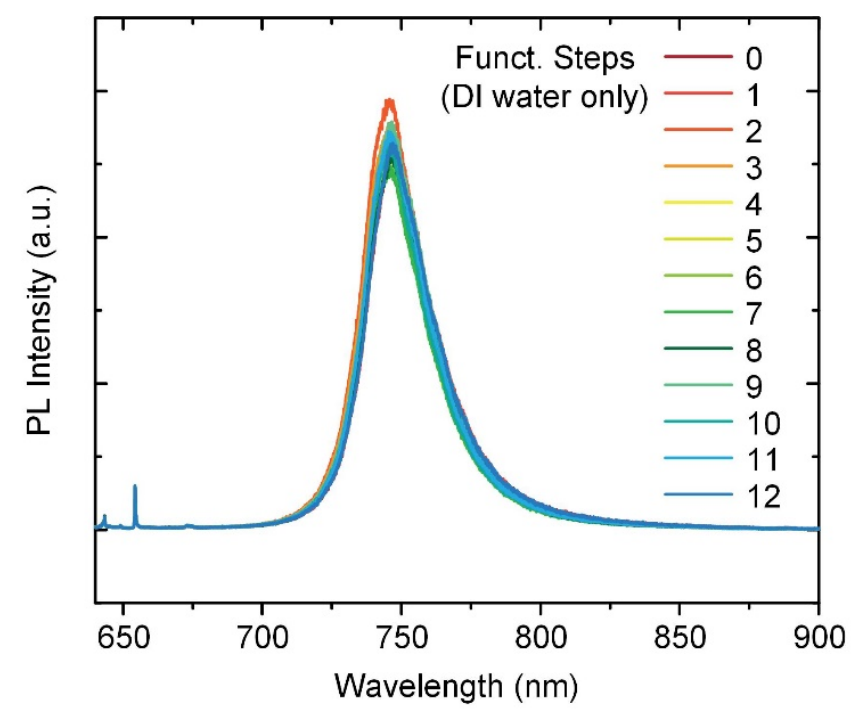

Figure S14. Monolayer WSez was annealed and immersed in DI water for 5 min without NiPc molecules for each step, which is identical to the functionalization steps presented in Figure 4a. The PL does not quench throughout the experiments, indicating negligible effects of DI water.

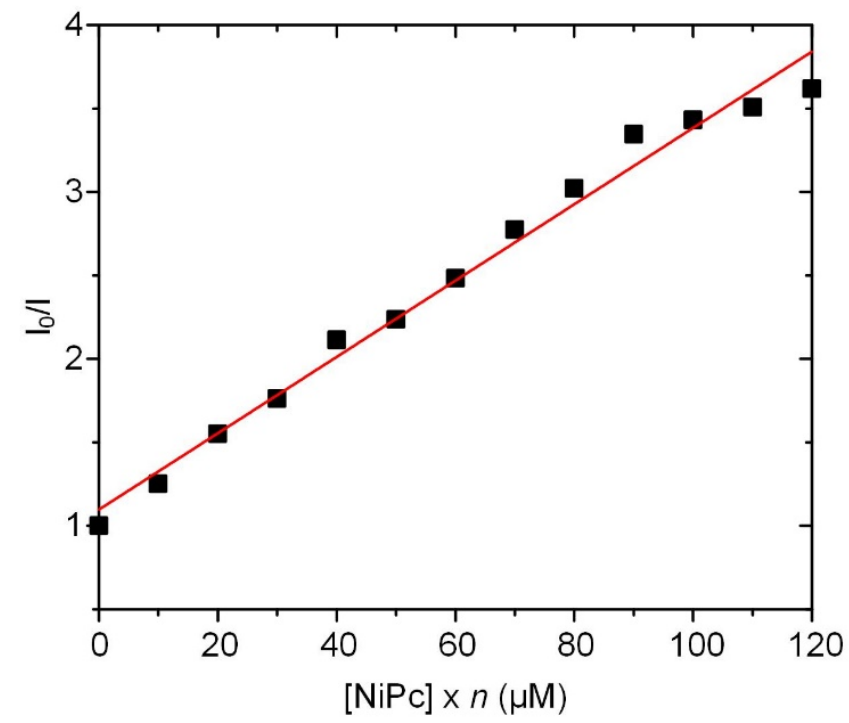

Figure S15. WSe2 PL quenching with increasing functionalization step. We assumed that the NiPc concentration increases linearly with the number of functionalization steps. For each functionalization step, the WSe2 was immersed in NiPc solution $([\mathrm{NiPc}]=10 \mu \mathrm{M})$ for $5 \mathrm{~min}$, followed by DI rinsing and drying. The quenching behavior follows a static quenching equation, $I_{0} / I=1+K n$ [MPc], where $I$ and $I_{o}$ are the PL intensity with and without quencher (NiPc in this case), $K$ is the association constant, and $n$ is the number of functionalization step. The association constant $\left(K=2.3 \times 10^{4} \mathrm{M}^{-1}\right)$ can be estimated from the linear relation between the $I_{0} / I$ and the increasing functionalization step of NiPc. 


\section{Analysis of $I-V$ curves measured using PC-AFM}

We measured the current transport behaviors of WSe2 and WSe2-NiPc in dark and under illumination using PC-AFM. The experimental data was fitted to the thermionic emission model ${ }^{15}$ as presented in Figure $5 \mathrm{~b}$ and $\mathrm{c}$ in the main text. The current $I$ and saturation current $I 0$ are given by:

$$
\begin{aligned}
I & =I_{0}\left[\exp \left(\frac{q V}{\eta k_{B} T}\right)-1\right] \\
I_{0} & =A_{e} A^{*} T^{2} \exp \left(-\frac{q \Phi_{B}}{k_{B} T}\right)
\end{aligned}
$$

where $q$ is the electronic charge, $V$ is the applied bias voltage, $\eta$ is the ideality factor, $k_{B}$ is the Boltzmann constant, $\Phi_{B}$ is the barrier height, $A_{e}$ is the effective contact area, $A^{*}$ is the Richardson constant, and $T$ is the temperature. From the contact force $(\sim 20 \mathrm{nN})$ that we used during the PCAFM measurements, the effective contact area $\left(A_{e}\right)$ was estimated to be roughly $5 \mathrm{~nm}^{2} .{ }^{16}$ The Richardson constant $\left(A^{*}\right)$ can be expressed as:

$$
A^{*}=\frac{4 \pi q m^{*} k_{B}^{2}}{h^{3}}
$$

where $m^{*}$ is the effective electron mass and $h$ is the Planck constant. For free electrons $\left(m^{*}=m_{0}\right.$, where $m_{0}$ is the electron mass), the Richardson constant is $120 \mathrm{~A} \mathrm{~cm}^{-2} \mathrm{~K}^{-2}{ }^{15}$ The ideality factor $(\eta)$ can be estimated from the slope of the linear relation in $d V / d(\ln I)$ :

$$
\frac{1}{\eta}=\frac{k_{B} T}{q} \frac{d(\ln I)}{d V}
$$

and the saturation current $\left(I_{0}\right)$ can be obtained by fitting the experimental data to the thermionic emission model. Finally, we can calculate the barrier height from the equation below:

$$
\Phi_{B}=\frac{k_{B} T}{q} \ln \left(\frac{A_{e} A^{*} T^{2}}{I_{0}}\right)
$$

Estimated barrier height from the thermionic emission model is 0.17 and $0.21 \mathrm{eV}$ for forward and reverse bias in dark, and 0.15 and $0.18 \mathrm{eV}$ for forward and reverse bias under illumination. After NiPc functionalization, the barrier height is estimated to be 0.18 and $0.21 \mathrm{eV}$ for forward and reverse bias in dark, and 0.13 and $0.12 \mathrm{eV}$ for forward and reverse bias under illumination. The obtained barrier height is slightly smaller than the value obtained from the band alignment $(\sim 0.2 \mathrm{eV})$ in Figure S16a, possibly due to tunneling current which is not considered in the thermionic model. ${ }^{16}$ Nevertheless, the decreased barrier height under illumination corresponds to the increased current flow by the contribution of photo-generated carriers, and it is more significant when the WSez is functionalized with NiPc which facilitates charge separation.

The fitting results are plotted in semi-log $I-V$ curves as shown in Figure 16b, which shows typical p-type transport behavior. ${ }^{11}$ In dark condition, the $I-V$ curves are similar before and after $\mathrm{NiPc}$ functionalization, indicating the NiPc does not disturb current flow. However, under 
illumination, the NiPc-functionalized WSe2 shows improved photocurrent than the pristine WSe2, as clearly seen in Figure 16b.

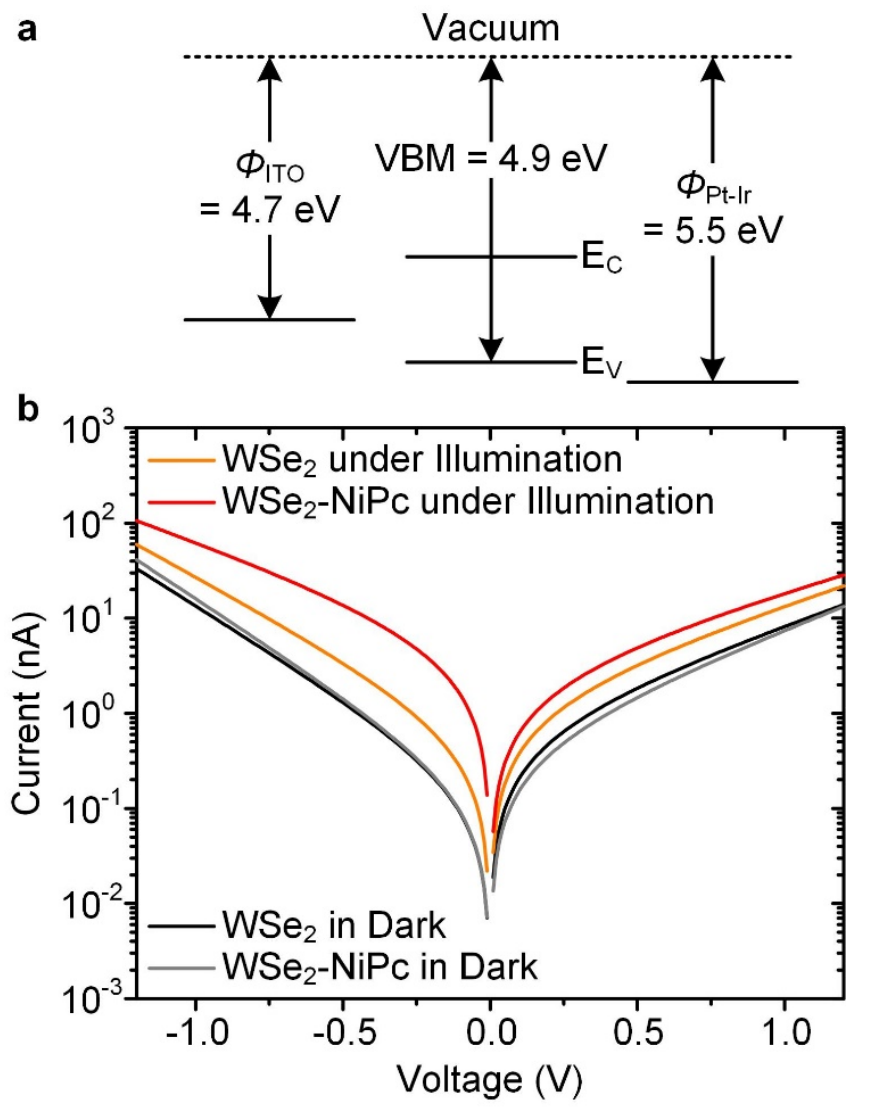

Figure S16. (a) Energy band diagram of ITO/WSe $2 / \mathrm{Pt}$-Ir, where $\Phi_{\text {ITO }}$ is the work function of ITO (4.7 eV), VBM of WSe2 (valence band maximum, $\sim 4.9 \mathrm{eV}$ ), ${ }^{17}$ and $\Phi_{\mathrm{Pt}-\mathrm{Ir}}$ is the work function of Pt-It probe $(5.5 \mathrm{eV})$. Because $\Phi_{\mathrm{Pt}-\mathrm{Ir}}$ is aligned with the valence band of p-type $\mathrm{WSe}_{2}{ }^{18}$ it forms an ohmic contact, while the Schottky barrier forms between the ITO and the WSe2. The barrier height can be represented as $\Phi_{\mathrm{B}}=\mathrm{VBM}-\Phi_{\mathrm{ITO}}$ and calculated to be $\sim 0.2 \mathrm{eV}$. (b) Semi-log $I-V$ plots of $\mathrm{WSe}_{2}$ and WSe2-NiPc in dark and under illumination. The each curve is obtained from the fitting result of thermionic emission model. 


\section{2D current profiles and PL spectra of WSe ${ }_{2}$ and NiPc-functionalized $W_{S} e_{2}$}
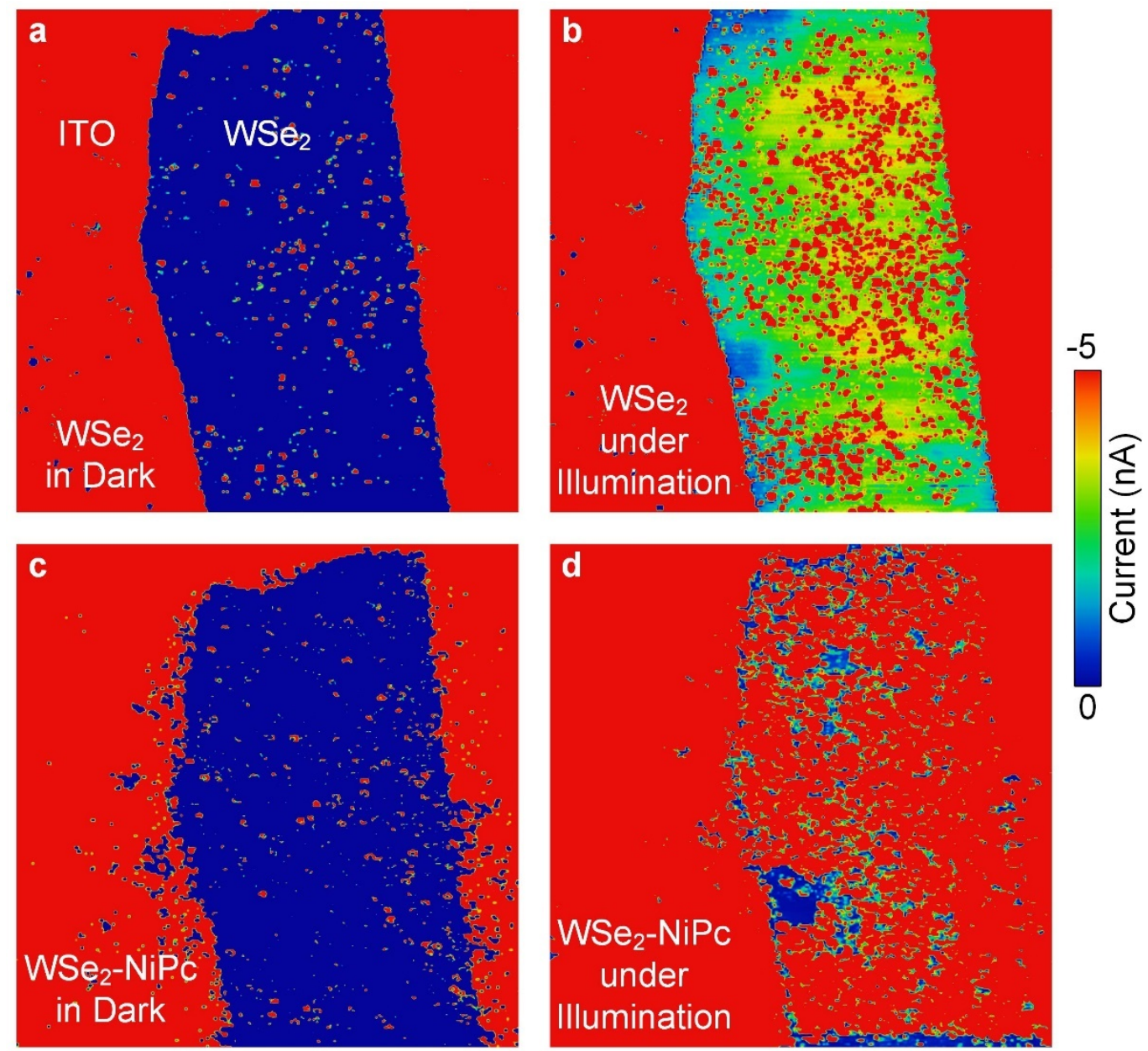

Figure S17. 2D current profiles of (a) WSe2 in dark, (b) WSe2 under illumination ( $\lambda_{\mathrm{ex}}=658 \mathrm{~nm}$ ), (c) WSe2-NiPc in dark, and (d) WSe2-NiPc under illumination $\left(\lambda_{\text {ex }}=658 \mathrm{~nm}\right)$. The raster-scanned current profiles were measured by PC-AFM at fixed bias voltage of $-0.5 \mathrm{~V}$. These images are used to reconstruct the photocurrent maps by subtracting (a) from (b) for Figure 5e and (c) from (d) for Figure $5 \mathrm{f}$ in the main text. 


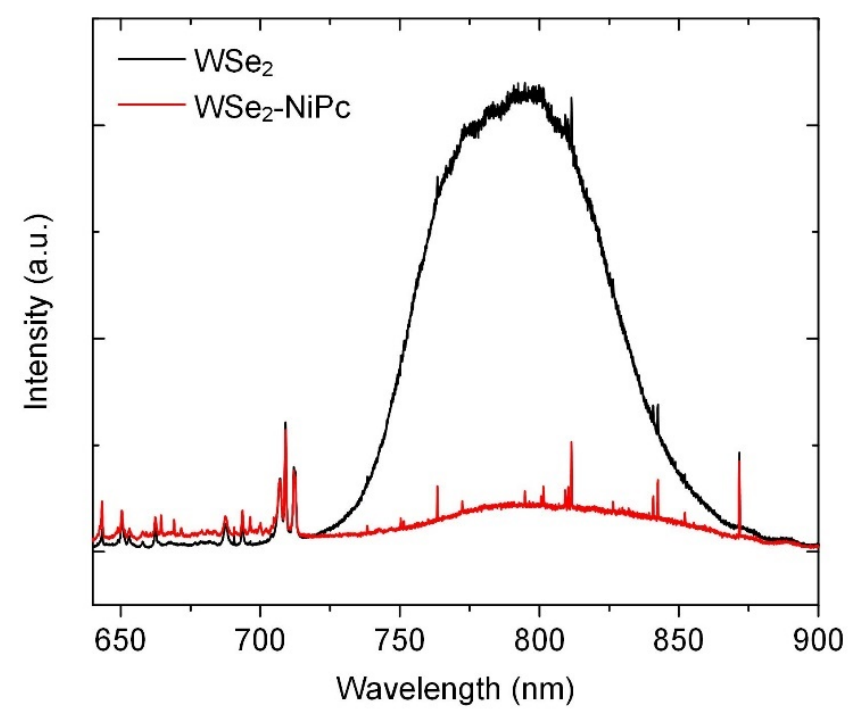

Figure S18. PL spectra of (a) pristine WSe 2 and (b) NiPc-functionalized WSe 2 exfoliated on the ITO substrate. A few Raman signals from the ITO are also shown. Significant PL quenching is observed as expected. The PL quenching is originated from excited electron transfer from the WSe2 layer to the NiPc molecules, which also contributes to the increased photocurrent after functionalization. It is noted that the spectra were taken from the identical WSe2 presented in Figure $5 \mathrm{~d}-\mathrm{f}$ and Figure S17. 


\section{Photocurrent maps and PL spectra of MPc-functionalized TMDCs}
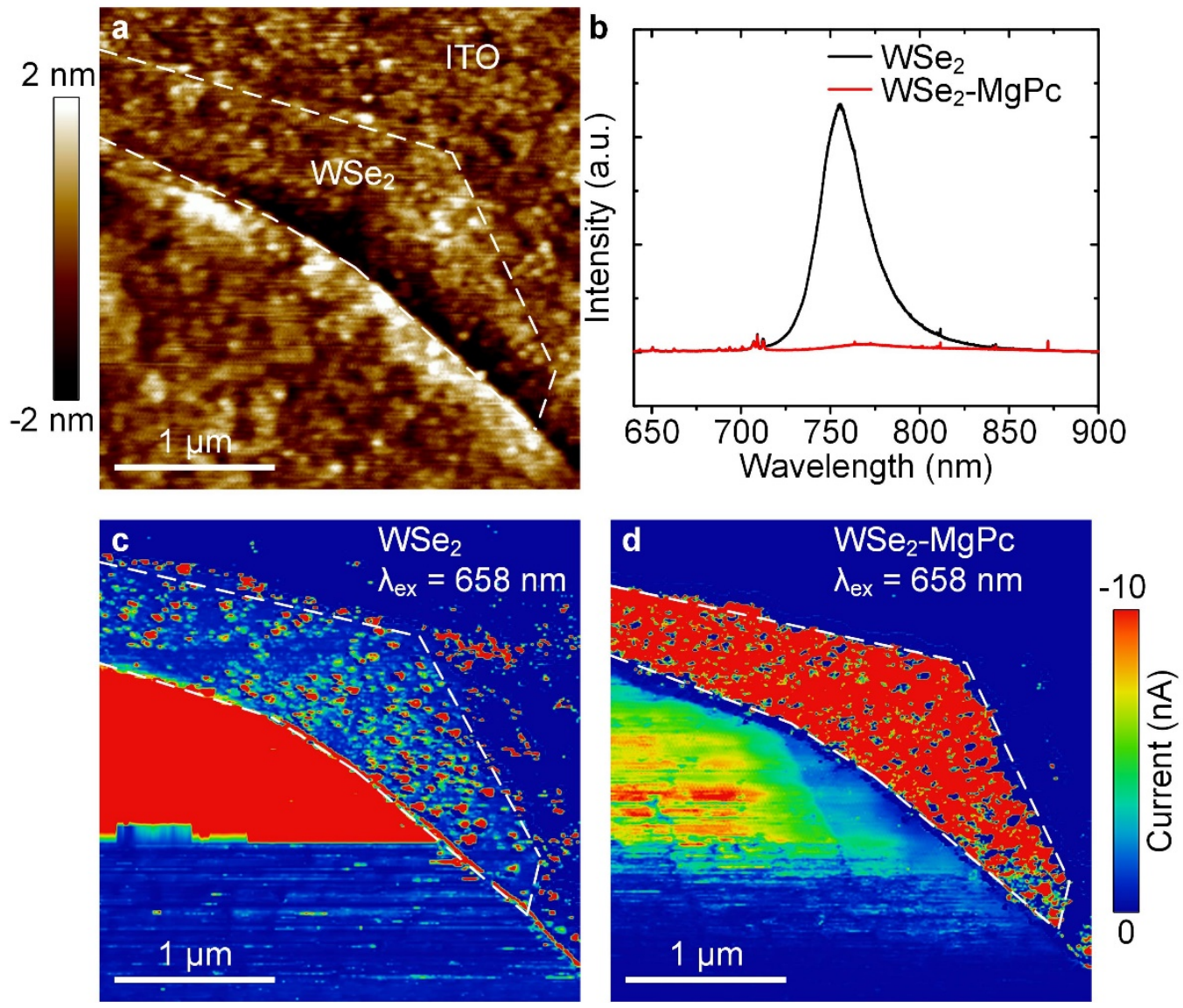

Figure S19. Characterization of WSez before and after MgPc functionalization. (a) AFM height image of WSe2 exfoliated on an ITO substrate. (b) PL spectra before and after MgPc functionalization, which shows significant PL quenching after functionalization due to excited electron transfer. (c) Photocurrent map of pristine WSe2 under illumination of $658 \mathrm{~nm}$ with a bias voltage of $-0.4 \mathrm{~V}$. An average photocurrent is $-2.4 \pm 0.9 \mathrm{nA}$. (d) Photocurrent map of MgPcfunctionalized WSe2 measured under the same conditions as the pristine WSe2. A drastic increase of photocurrent is observed with an average of $-15.6 \pm 1.9 \mathrm{nA}$. The PL quenching and increased photocurrent after MgPc functionalization on WSe2 can be explained by the photoinduced charge transfer mechanism. 

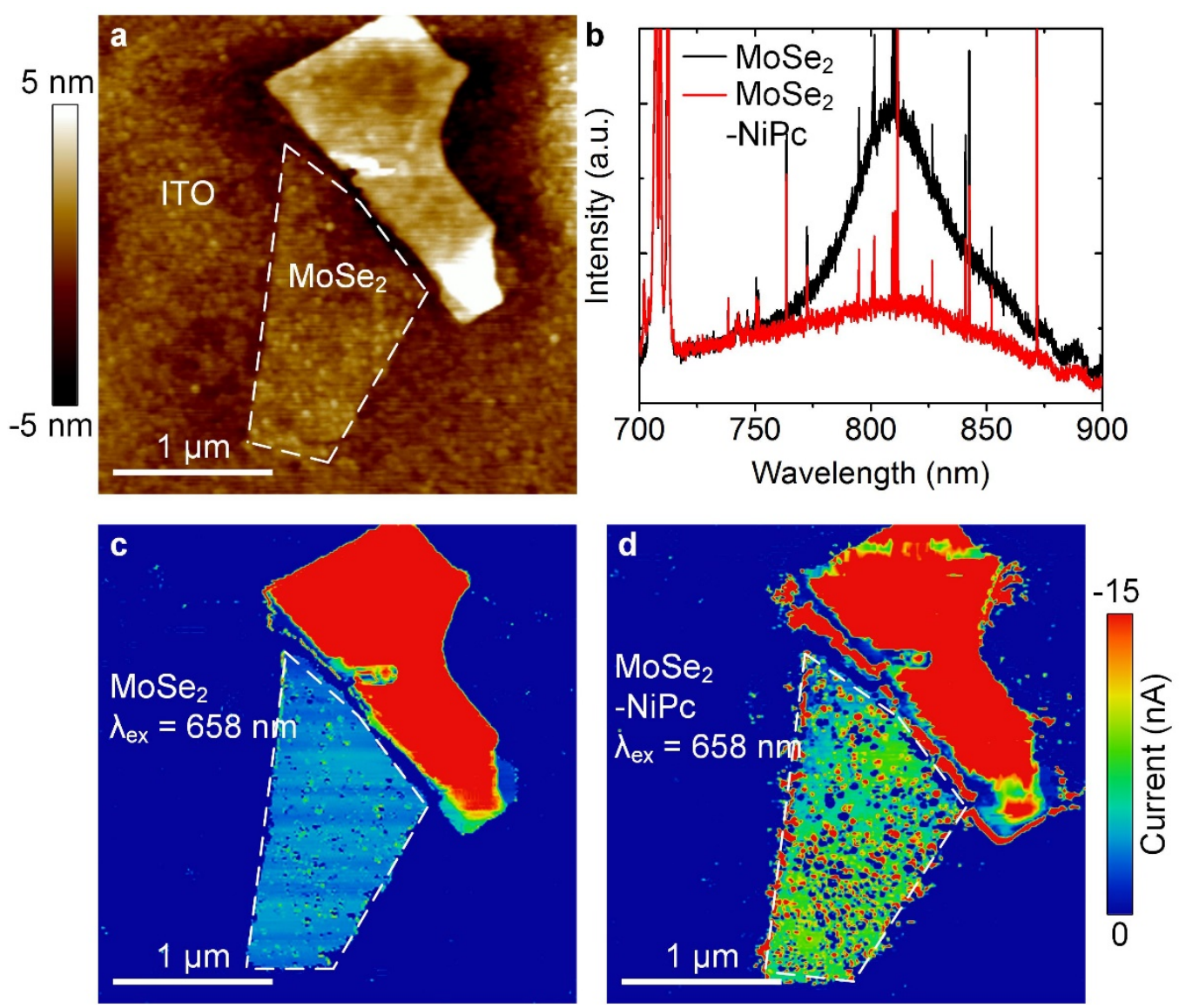

Figure S20. Characterization of MoSez before and after NiPc functionalization. (a) AFM height image of MoSez exfoliated on the ITO substrate. (b) PL spectra before and after NiPc functionalization, which shows PL quenching after functionalization due to excited electron transfer. (c) Photocurrent map of pristine MoSe2 under illumination of $658 \mathrm{~nm}$ with a bias voltage of $-0.4 \mathrm{~V}$. An average photocurrent is $-4.8 \pm 0.5 \mathrm{nA}$. (d) Photocurrent map of NiPc-functionalized $\mathrm{MoSe}_{2}$ measured under the same conditions as the pristine MoSe2. An increase of photocurrent is observed with an average of $-6.3 \pm 1.8 \mathrm{nA}$. The PL quenching and increased photocurrent after $\mathrm{NiPc}$ functionalization on $\mathrm{MoSe}_{2}$ can be explained by the photoinduced charge transfer mechanism. 

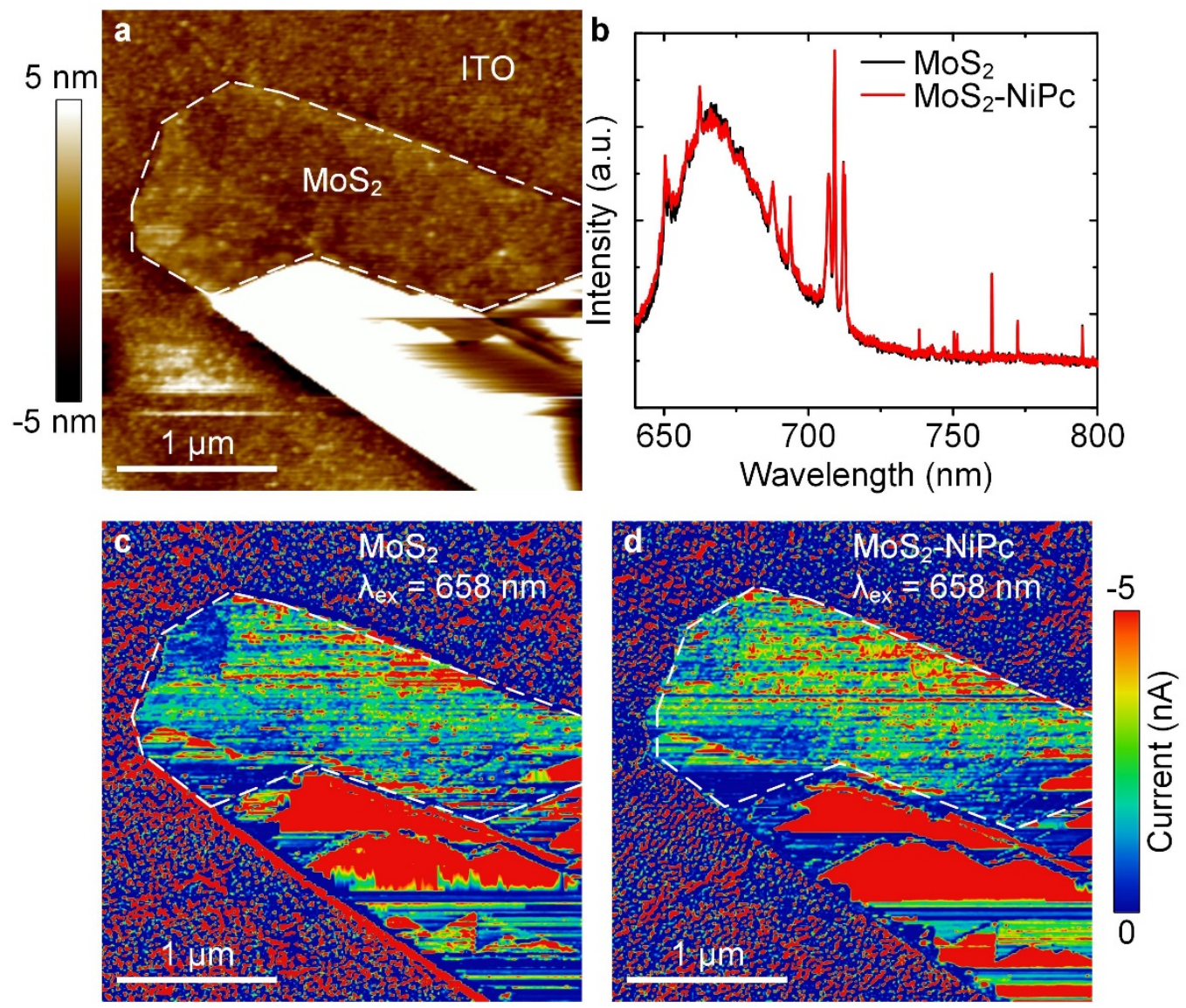

Figure S21. Characterization of $\mathrm{MoS}_{2}$ before and after NiPc functionalization. (a) AFM height image of $\mathrm{MoS}_{2}$ exfoliated on the ITO substrate. (b) No significant quenching in PL spectra is observed before and after NiPc functionalization on $\mathrm{MoS}_{2}$. (c) Photocurrent map of pristine $\mathrm{MoS}_{2}$ under illumination of $658 \mathrm{~nm}$ with a bias voltage of $-0.4 \mathrm{~V}$. An average photocurrent is $-2.3 \pm 0.9$ nA. (d) Photocurrent map of NiPc-functionalized $\mathrm{MoS}_{2}$ measured under the same conditions as the pristine $\mathrm{MoS}_{2}$. The photocurrent remains nearly the same as that of pristine $\mathrm{MoS}_{2}$ with an average of $-2.4 \pm 1.1 \mathrm{nA}$. This observation is consistent with the photoinduced charge transfer mechanism, where excited electrons from the $\mathrm{MoS}_{2}$ are not allow to transfer to the NiPc. 


\section{References}

1. Tongay, S.; Zhou, J.; Ataca, C.; Liu, J.; Kang, J. S.; Matthews, T. S.; You, L.; Li, J.; Grossman, J. C.; Wu, J. Broad-Range Modulation of Light Emission in Two-Dimensional Semiconductors by Molecular Physisorption Gating. Nano Lett, 2013, 13, 2831-2836.

2. Nan, H.; Wang, Z.; Wang, W.; Liang, Z.; Lu, Y.; Chen, Q.; He, D.; Tan, P.; Miao, F.; Wang, X.; Wang, J.; Ni, Z. Strong Photoluminescence Enhancement of $\mathrm{MoS}_{2}$ through Defect Engineering and Oxygen Bonding. ACS Nano 2014, 8, 5738-5745.

3. Mouri, S.; Miyauchi, Y.; Matsuda, K. Tunable Photoluminescence of Monolayer MoS 2 via Chemical Doping. Nano Lett. 2013, 13, 5944-5948.

4. Peimyoo, N.; Yang, W.; Shang, J.; Shen, X.; Wang, Y.; Yu, T. Chemically Driven Tunable Light Emission of Charged and Neutral Excitons in Monolayer WS 2 . ACS Nano 2014, 8, 11320-11329.

5. Lin, J. D.; Han, C.; Wang, F.; Wang, R.; Xiang, D.; Qin, S.; Zhang, X.-A.; Wang, L.; Zhang, H.; Wee, A. T. S.; Chen, W. Electron-Doping-Enhanced Trion Formation in Monolayer Molybdenum Disulfide Functionalized with Cesium Carbonate. ACS Nano 2014, 8, 53235329.

6. Tongay, S.; Suh, J.; Ataca, C.; Fan, W.; Luce, A.; Kang, J. S.; Liu, J.; Ko, C.; Raghunathanan, R.; Zhou, J.; Ogletree, F.; Li, J.; Grossman, J. C.; Wu, J. Defects Activated Photoluminescence in Two-Dimensional Semiconductors: Interplay between Bound, Charged, and Free Excitons. Sci. Rep. 2013, 3, 2657.

7. de Faria, D. L. A.; Constantino, V. R. L.; Baldwin, K. J.; Batchelder, D. N.; Pinnavaia, T. J.; Chibwe, M. Raman Microspectroscopy of Phthalocyanine Intercalates: Ttetrasulphonated Cobalt and Nickel Phthalocyanines in Layered Double Hydroxide. J. Raman Spectrosc. 1998, 29, 103-108.

8. Kahl, J. L.; Faulkner, L. R.; Dwarakanath, K.; Tachikawa, H. Reversible Oxidation and Rereduction of Magnesium Phthalocyanine Electrodes. Electrochemical Behavior and in situ Raman Spectroscopy. J. Am. Chem. Soc. 1986, 108, 5434-5440.

9. Yang, L.; Majumdar, K.; Liu, H.; Du, Y.; Wu, H.; Hatzistergos, M.; Hung, P. Y.; Tieckelmann, R.; Tsai, W.; Hobbs, C.; Ye, P. D. Chloride Molecular Doping Technique on 2D Materials: $\mathrm{WS}_{2}$ and $\mathrm{MoS}_{2}$. Nano Lett. 2014, 14, 6275-6280.

10. Fang, H.; Tosun, M.; Seol, G.; Chang, T. C.; Takei, K.; Guo, J.; Javey, A. Degenerate n-Doping of Few-Layer Transition Metal Dichalcogenides by Potassium. Nano Lett. 2013, 13, 19911995.

11. McDonnell, S.; Addou, R.; Buie, C.; Wallace, R. M.; Hinkle, C. L. Defect-Dominated Doping and Contact Resistance in MoS2. ACS Nano 2014, 8, 2880-2888.

12. McDonnell, S.; Azcatl, A.; Addou, R.; Gong, C.; Battaglia, C.; Chuang, S.; Cho, K.; Javey, A.; Wallace, R. M. Hole Contacts on Transition Metal Dichalcogenides: Interface Chemistry and Band Alignments. ACS Nano 2014, 8, 6265-6272.

13. Montalti, M.; Credi, A.; Prodi, L.; Gandolfi, M. T. Handbook of Photochemistry, 3rd ed.; CRC Press: Boca Raton, Florida, 2006.

14. Zhao, W.; Ribeiro, R. M.; Toh, M.; Carvalho, A.; Kloc, C.; Castro Neto, A. H.; Eda, G. Origin of Indirect Optical Transitions in Few-Layer $\mathrm{MoS}_{2}, \mathrm{WS}_{2}$, and WSe2. Nano Lett. 2013, 13, 5627-5634.

15. Sze, S. M.; Ng, K. K. Physics of Semiconductor Devices, 3rd ed.; Wiley-Interscience: Hoboken, New Jersey, 2007. 
16. Son, Y.; Wang, Q. H.; Paulson, J. A.; Shih, C.-J.; Rajan, A. G.; Tvrdy, K.; Kim, S.; Alfeeli, B.; Braatz, R. D.; Strano, M. S. Layer Number Dependence of $\mathrm{MoS}_{2}$ Photoconductivity Using Photocurrent Spectral Atomic Force Microscopic Imaging. ACS Nano 2015, 9, 2843-2855.

17. Gong, C.; Zhang, H.; Wang, W.; Colombo, L.; Wallace, R. M.; Cho, K. Band Alignment of Two-Dimensional Transition Metal Dichalcogenides: Application in Tunnel Field Effect Transistors. Appl. Phys. Lett. 2013, 103, 053513.

18. Fang, H.; Chuang, S.; Chang, T. C.; Takei, K.; Takahashi, T.; Javey, A. High-Performance Single Layered WSe2 p-FETs with Chemically Doped Contacts. Nano Lett. 2012, 12, 37883792. 